\title{
Tie2 as a novel key factor of microangiopathy in systemic sclerosis
}

\author{
Falk Moritz ${ }^{1,2}$, Janine Schniering ${ }^{1}$, Jörg H. W. Distler ${ }^{3}$, Renate E. Gay ${ }^{1}$, Steffen Gay ${ }^{1}$, Oliver Distler ${ }^{1}$ \\ and Britta Maurer ${ }^{1 *}$
}

\begin{abstract}
Background: The angiopoietin(Ang)/Tie2 system is a key regulator of vascular biology. The expression of membrane bound (mb) Tie2 and Ang-1 ensures vessel stability, whereas Ang-2, inducible by vascular endothelial growth factor (VEGF), hypoxia, and inflammation, acts as an antagonist. Tie2 signalling is also attenuated by soluble Tie2 (sTie2), the extracellular domain of the receptor, which is shed upon stimulation with VEGF. Herein, we investigate the role of Ang/Tie2 in the peripheral vasculopathy in systemic sclerosis (SSC) including animal models.

Methods: The expression of Ang-1/-2 and Tie2 in skin/serum of SSc patients was compared with healthy controls by immunohistochemistry (IHC)/ELISA. Expression of Ang/Tie2 was analysed in different animal models: VEGF transgenic (tg) mice, hypoxia model, bleomycin-induced skin fibrosis, and tight skin 1 (TSK1) mice.

Results: In SSc, dermal microvessels abundantly expressed Ang-2, but not Ang-1 compared with healthy controls. The percentage of mbTie2+ microvessels was profoundly decreased whereas the levels of sTie2 were increased already in early disease. Both in skin and sera of SSc patients, the Ang1/2 ratio was reduced, being lowest in patients with digital ulcers indicating vessel destabilizing conditions. We next studied potential influencing factors in animal models. The VEGF tg mouse model, the hypoxia, and the inflammation-dependent bleomycin model all showed a similar dysregulation of Ang/Tie2 as in SSc, which did not apply for the non-inflammatory TSK1 model.

Conclusion: Peripheral microvasculopathy in SSc results from a complex dysregulation of angiogenic signalling networks including the VEGF and the Ang/Tie2 system. The profoundly disturbed Ang-/Tie-2 balance might represent an important target for vascular therapeutic approaches in SSc.
\end{abstract}

Keywords: Microvasculopathy, Systemic sclerosis, Angiopoietins, Tie2

\section{Background}

Microvascular injury due to dysregulation of angiogenic factors such as vascular endothelial growth factor (VEGF) and hypoxia [1-4] is a very early pathogenic event in systemic sclerosis (SSc) [5]. The Angiopoieti$\mathrm{n}$ (Ang)/Tie2 system is a key regulator of angiogenesis. The binding of Ang-1, produced by vascular smooth muscle cells and other perivascular cells, to the membrane-bound $(\mathrm{mb})$ Tie2 receptor on endothelial cells (EC) is crucial for vessel stability. Tie2 activation promotes vessel assembly and maturation by mediating survival signals for EC and regulating the recruitment of pericytes [6]. Ang-2, inducible

\footnotetext{
*Correspondence: britta.maurer@usz.ch

'Department of Rheumatology, University Hospital Zurich, Gloriastrasse 25, 8091 Zurich, Switzerland

Full list of author information is available at the end of the article
}

in EC by VEGF, hypoxia, and inflammation [6-8], antagonizes the Ang-1/Tie2 pathway by competing with Ang-1. Both ligands bind to Tie 2 with similar affinity. Under physiologic conditions, the levels of Ang-1 exceed those of Ang-2, thereby ensuring the quiescent state of the vasculature. Increased levels of Ang-2 are associated with vascular remodelling [9]. Furthermore, the effects of Ang-2 are VEGF-dependent. In the absence of VEGF, Ang-2 has vessel destabilizing effects and induces regression of microvessels $[10,11]$. In the presence of VEGF, Ang-2 induces angiogenesis with endothelial cell proliferation, migration and disruption of the vascular basement membrane due to the increased expression of matrix metalloproteinases [12]. Thus, the Ang-1/-2 ratio determines the functional status of the local vasculature. In contrast to mbTie2, little 
is known about soluble Tie2 (sTie2), the extracellular domain of the Tie2 receptor, which is released by proteolytic cleavage by matrix metalloproteases upon stimulation with VEGF in a process also referred to as shedding $[13,14]$. sTie 2 is detectable in healthy individuals, and increased serum concentrations were measured in cardiovascular diseases, diabetic retinopathy [15], and SSc [16, 17].

Previous studies supported an equal affinity of Ang-1 and Ang-2 for the Tie2 receptor [10, 11, 13]. Interestingly, a recent study reported a higher affinity of Ang-1 to sTie2, which indicates that sTie2 might shift the Ang-1/Ang-2 ratio in favour of Ang-2 thereby inducing destabilization and regression of microvessels [18]. Other studies suggest competitive binding to mbTie2 thereby blocking access for Ang-1/-2 with inhibition of downstream signalling [6]. The scheme in Fig. 1 summarizes the current concept of the role of Ang/Tie2 in vascular remodelling.
In the present study, we assessed alterations of the Ang/Tie2 system in SSc-associated dermal microvasculopathy and in different animal models of SSc.

\section{Methods \\ Patients}

Serum samples were obtained from patients with limited cutaneous (lc) SSc $(n=21)$ and diffuse cutaneous (dc) SSc patients $(\mathrm{n}=13)$ fulfilling the LeRoy criteria [19], patients with early SSc not yet fulfilling the LeRoy criteria $(=$ pre-SSc) $(n=12)$, and from age- and sex-matched healthy donors $(n=40)$. The patients were $56.2 \pm 11.6$ years old, the majority was female $(75 \%)$. The average disease duration was $6.3 \pm 4.64$ years. All patients had Raynaud's phenomenon, $68 \%$ had a history of digital ulcers, $37 \%$ had current digital ulcers, and 93\% had teleangiectasias. The majority of patients were ANA positive (88.3\%). A total of $39 \%$ were positive for anti-Scl-70, $44 \%$ for anti-centromere, and $2 \%$ for anti-

\section{A Quiescent state}
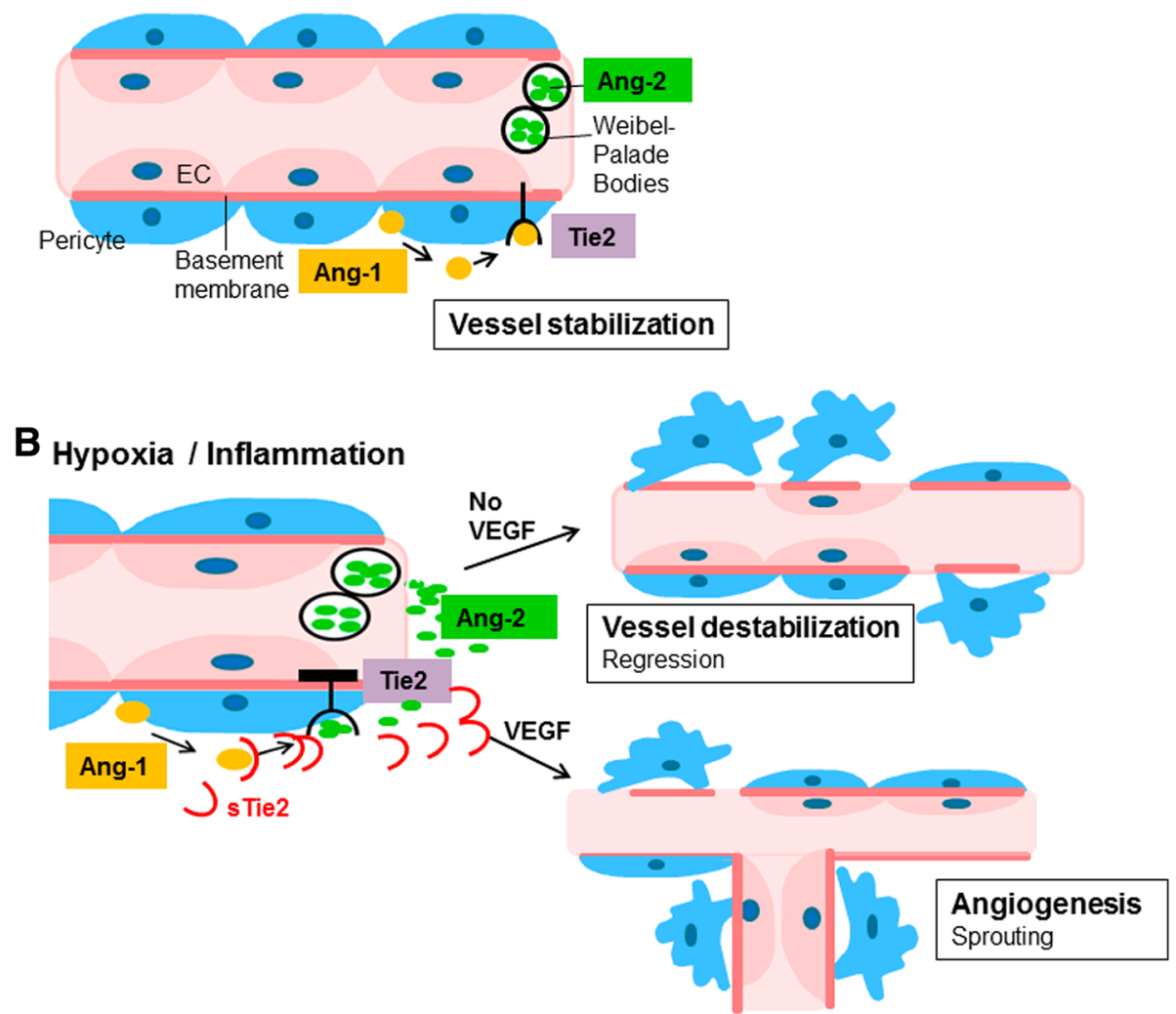

Fig. 1 The role of Angiopoietins and Tie2 in vascular biology (adapted from [6-8, 11, 13, 14]). a In quiescent state, Ang-1, produced by pericytes, acts in a paracrine manner on the membrane-bound $(\mathrm{mb})$ Tie2 receptor on endothelial cells (EC). The effects of Ang-1/Tie2 signalling ensure vessel stabilization. Ang-2, produced by EC, is stored in Weibel-Palade bodies. $\mathbf{b}$ In hypoxia and inflammation, Ang-2 is released from the WeibelPalade bodies and acts in an autocrine manner on EC. Due to competitive binding to mbTie2, Ang-2 antagonizes Ang- 1 signalling. In the absence of VEGF, the inhibition of Tie2 signalling leads to vessel destabilization and regression due to apoptosis of EC, loss of pericyte coverage and disrupture of the basement membrane. In the presence of VEGF, Ang-2 exerts pro-angiogenic effects with proliferation and migration of EC and sprouting of new branches. Additionally, VEGF causes shedding of Tie2. sTie acts as competitive ligand for Ang-1/-2 thereby blocking downstream signalling with anti-angiogenic effects. Ang Angiopoietin, VEGF vascular endothelial growth factor 
RNA-polymerase III. The majority of patients had an active pattern in the nailfold capillaroscopy, whereas $33 \%$ showed a late, and $17 \%$ an early, pattern [20]. Skin biopsies were obtained by punch biopsies from involved skin at the forearms from 24 of the above mentioned SSc patients (13 lcSSc, $11 \mathrm{dcSSc}$ ) and from 19 of the above mentioned healthy controls. All participants had signed a consent form approved by the local institutional review boards. The study had been approved by the cantonal ethics committee Zurich.

\section{Animals \\ VEGF tg mice}

To investigate the effect of VEGF on Ang/Tie2, VEGF homo- $(+/+) \quad(n=8)$ and heterozygous $(+/-) \quad(n=9)$ transgenic $(\mathrm{tg})$ mice and wildtype (wt) littermates $(n=$ 6/9) were analysed. VEGF tg mice have been described previously [21]. Briefly, in VEGF tg mice, the murine VEGF164 gene which is the murine equivalent to VEGF-A165, was cloned into a human keratin 14 promoter expression cassette which had previously been shown to selectively target transgene expression to basal keratinocytes of the skin [21]. VEGF tg mice were on the FVB background.

\section{Hypoxia model}

Four- to six-week-old female C57BL/6 mice $(\mathrm{n}=3)$ were exposed to systemic normobaric hypoxia by substitution of oxygen with nitrogen in a closed Perspex chamber using a Digamix 2 M 302/a-F pump (H. Woesthoff $\mathrm{GmbH}$, Bochum, Germany) at a flow rate of $37 \mathrm{l} / \mathrm{min}$. When mice were placed into the chamber, $\mathrm{O}_{2}$ fractions were decreased gradually from 21 to $6 \%$ within $1 \mathrm{~h}$ and mice were kept in the hypoxic chamber for additional 24 to $48 \mathrm{~h}$. In parallel, nine littermates were kept under normoxic conditions. The experimental set-up was described previously [4].

\section{Bleomycin-induced skin fibrosis in mice}

The bleomycin model is a frequently used model of chemically induced dermal fibrosis. The bleomycininduced skin fibrosis in mice is considered as an animal model that mimics early, pro-inflammatory stages of skin fibrosis in SSc. Skin fibrosis was induced in 6- to 8-week-old C57/BL6 mice $(\mathrm{n}=4)$ by local injections of bleomycin for 21 days as previously described [22]. Briefly, $100 \mu \mathrm{l}$ of bleomycin dissolved in $0.9 \%$ sodium chloride $(\mathrm{NaCl})$ at a concentration of $0.5 \mathrm{mg} / \mathrm{ml}$ was administered every other day by subcutaneous injections in defined areas of the upper back. Subcutaneous injections of $100 \mu \mathrm{l} 0.9 \% \mathrm{NaCl}$ were used in a second group $(n=4)$ as controls.

\section{TSK1 mice}

Compared to the bleomycin model, tight skin 1 (TSK1) mice [23] are considered as a late-stage, non-inflammatory model of skin fibrosis in SSc. Due to a dominant mutation of the fibrillin 1 gene, TSK1 mice develop an increased dermal and especially hypodermal thickness. TSK1 mice were interbred with $\mathrm{pa} / \mathrm{pa}$ mice in which a recessive mutation (pa) induces a light grey colour of the fur and pink eyes. Because the fibrillin 1 gene is genetically linked to the pa gene, mice can be screened for the tsk 1 mutation based on the colour of eyes and fur. All mice with black fur and eyes carry the dominant tsk 1 mutation and are heterozygous for the pa mutation. In contrast, mice with grey fur and pink eyes do not carry the tsk1 mutation, but are homozygous for the mutated pa gene. Apart from changing the skin colour, the pa mutation does not alter skin physiology and has no impact on skin fibrosis [23]. In the current experiments, four TSK1 and four pa/pa mice were analysed.

The animal protocols were approved by the cantonal veterinary office Zurich.

\section{Immunohistochemistry}

Murine and human skin samples were fixed in $4 \%$ formalin and embedded into paraffin as described previously [24]. For immunohistochemistry, $5 \mu \mathrm{m}$ thick sections of human and murine skin were used. Immunohistochemical double stainings were performed in sequential order. On the first day, after pre-treatment with citrate buffer, the slides were incubated with primary rabbit polyclonal von Willebrand Factor (vWF) antibody (Abcam, Cambridge, UK) at room temperature for $1 \mathrm{~h}$, followed by incubation with biotin-labelled secondary goat anti-rabbit antibody (Jackson ImmunoResearch, Soham, UK). Staining was visualized with ABC phosphatase kit (Vector Laboratories, Burlingame, CA, USA) and developed using Vector blue. After additional heat-mediated treatment with citrate buffer, sections were incubated with primary rabbit polyclonal Ang-1 or Ang-2 antibodies (Abcam) overnight at $4{ }^{\circ} \mathrm{C}$, followed by incubation with biotin-labelled secondary goat antirabbit antibodies (Jackson ImmunoResearch). Stainings were visualized using an $\mathrm{ABC}$ peroxidase kit and developed using DAB (Vector Laboratories). The sequential staining with Tie2 was performed similarly with the exception that no additional heat-mediated removal was required on the second day since the primary Tie 2 antibody was mouse monoclonal (Abcam). Isotype-matched IgGs were used as negative controls.

\section{Analysis of skin sections}

The analyses of all immunohistochemical stainings were performed by two independent examiners who were blinded with regard to the different groups. All slides were analysed twice by each examiner. In case of 
a variation of the results $>10 \%$, the respective slides were re-assessed to reach consensus. Pictures were taken with a slide scanner (Zeiss Axio Scan.Z1), using Zen lite software (blue edition 2.3) (Carl Zeiss Microscopy GmbH, Jena, Germany).

For the semi-quantitative analysis of microvessel density, pictures of five randomly chosen high power fields (HPF)/slide were taken. Then, vWF+ blood vessels (dermal microvessels) were counted. Either the ratio or the percentage of Ang-1+, Ang-2+, or Tie2+ blood vessels was determined by assessing their numbers referred to the total number of vWF+ microvessels/HPF using the respective double stainings. Blood vessel density was assessed in the dermis without the epidermal and the subdermal layers, except for the TSK1 mice in which the hypodermis was analysed. The number/percentage of microvessels was then calculated for each group using GraphPad Prism software (GraphPad Software Inc., San Diego, CA, USA).

\section{ELISA}

Serum concentrations of Ang-1/-2 and sTie2 were measured using the colorimetric sandwich ELISA technique (Quantikine; R\&D Systems, Abingdon, UK).

\section{Real-time PCR}

Biopsy specimens $(0.5 \mathrm{~cm} 2)$ were homogenized with TissueLyser (Qiagen, Basel, Switzerland). Total RNA was isolated using the RNeasy Mini Kit (Qiagen, Hombrechtikon, Switzerland) and reverse transcribed into complementary DNA (cDNA) with random hexamers [25]. Expression of murine Ang-1, Ang-2, TEK (= Tie), and 18S rNA was measured using TaqMan Gene Expression Assays (Applied Biosystems, Basel, Switzerland). qPCR was performed using a 7500 Real-Time PCR System (Applied Biosystems). All qPCR experiments were performed in duplicate.

\section{Cell culture, reagents, stimulation assay, and analysis}

Human dermal microvascular endothelial cells (HMVEC$\mathrm{d}$ adult, Cambrex, Rockland, ME, USA) were cultured in six-well plates in endothelial cell medium (EGM-2 MV, Lonza, Basel, Switzerland), and passages 3-8 were used for analysis. After $24 \mathrm{~h}$ of serum reduction, HMVECs were stimulated for 12,24 , and $48 \mathrm{~h}$ with recombinant VEGFA165 (R\&D Systems) at concentrations of 2.5, 5, and $10 \mathrm{ng} / \mathrm{ml}$. Controls were exposed to equivalent dosages of the carrier protein BSA [26]. sTie was measured by ELISA as described above in cell culture supernatants. The percentage of Tie2+ cells was evaluated using flow cytometry analysis (FACS) according to the instructions of the manufacturer. Briefly, HMVECs were washed with PBS, detached with trypsin/0.5\% EDTA and centrifuged at $200 \times$ g for $5 \mathrm{~min}$. Afterwards cell pellets were resuspended and incubated for $15 \mathrm{~min}$ at room temperature with the primary Tie 2 antibody. Isotypematched IgGs were used as negative controls. The analysis was performed using the FACScan flow cytometer (Becton Dickinson, Mansfield, MA, USA). Data were analysed with CellQuest software (Becton Dickinson Immunocytometry Systems, San Jose, CA, USA).

\section{Statistical analysis}

For statistical analysis, GraphPad Prism software (version 5.01) was employed (GraphPad Software Inc.). Normal distribution of data was examined using the KolmogorovSmirnov test. For parametric non-related data, expressed as mean \pm standard error of the mean (SEM), the unpaired two-tailed $t$ test was used. Nonparametric non-related data, expressed as median ${ }_{(\mathrm{Q} 1, \mathrm{Q})}$ were analysed employing the Mann-Whitney $U$ test. $P$ values less than 0.05 were considered statistically significant.

Information on the histology and assessment of skin fibrosis in the bleomycin model and the measurement of collagen contents by hydroxyproline assay is provided in Additional file 1 supplemental methods.

\section{Results \\ Dysregulation of the Ang/Tie2 system in the dermal microvasculopathy in SSC}

In skin biopsies of SSc patients, the microvascular density was generally reduced compared with healthy controls (ratio of vWF+ vessels/HPF $27.3_{(24,38)}$ vs. $45.5_{(31,60)}$; $p=0.001$; Fig. 2a, b). Dermal capillaries of SSc patients expressed Ang-2 more abundantly than healthy controls (Fig. 2a, b) (ratio of Ang-2+/vWF+ microvessels/HPF $1.7_{(1.3,2.5)}$ vs. $\left.1_{(0.8,1.3)} ; p=0.01\right)$. Most impressively, the percentage of mbTie2-positive microvessels was strongly and consistently decreased in the skin of SSc patients compared with healthy controls (median (quartile range) $3.8_{(1,6)} \%$ vs. $87_{(78,99)} \%$; $p<0.001$ ) (Fig. 2a, b). The expression of Ang-1 did not differ in dermal capillaries of SSc patients compared with healthy controls (Fig. 2a). In the analysed sections, no difference in the expression of Ang-1/-2 or Tie2 could be observed between lcSSc or dcSSc. Furthermore, to evaluate whether the changes on tissue level were reflected on systemic level, we additionally measured the serum levels of Ang-1, Ang-2, and sTie2. To evaluate whether the loss of mbTie2 in dermal microvessels might be due to shedding [13], we assessed the serum levels of sTie2, which is the soluble, cleaved, extracellular domain of the Tie2 receptor. Indeed, sTie2 levels of SSc patients were higher than those of healthy controls (mean \pm SEM $9.6 \pm 1.7 \mathrm{ng} / \mathrm{ml}$ ) without differences between the lcSSc or dcSSc subsets $(16.7 \pm 7.5, \quad 13.6 \pm 1.7 \mathrm{ng} / \mathrm{ml} ; p<0.01) \quad$ (Fig. 3a). In accordance with the shedding hypothesis, treatment of human dermal microvascular endothelial cells (HMVECs) 


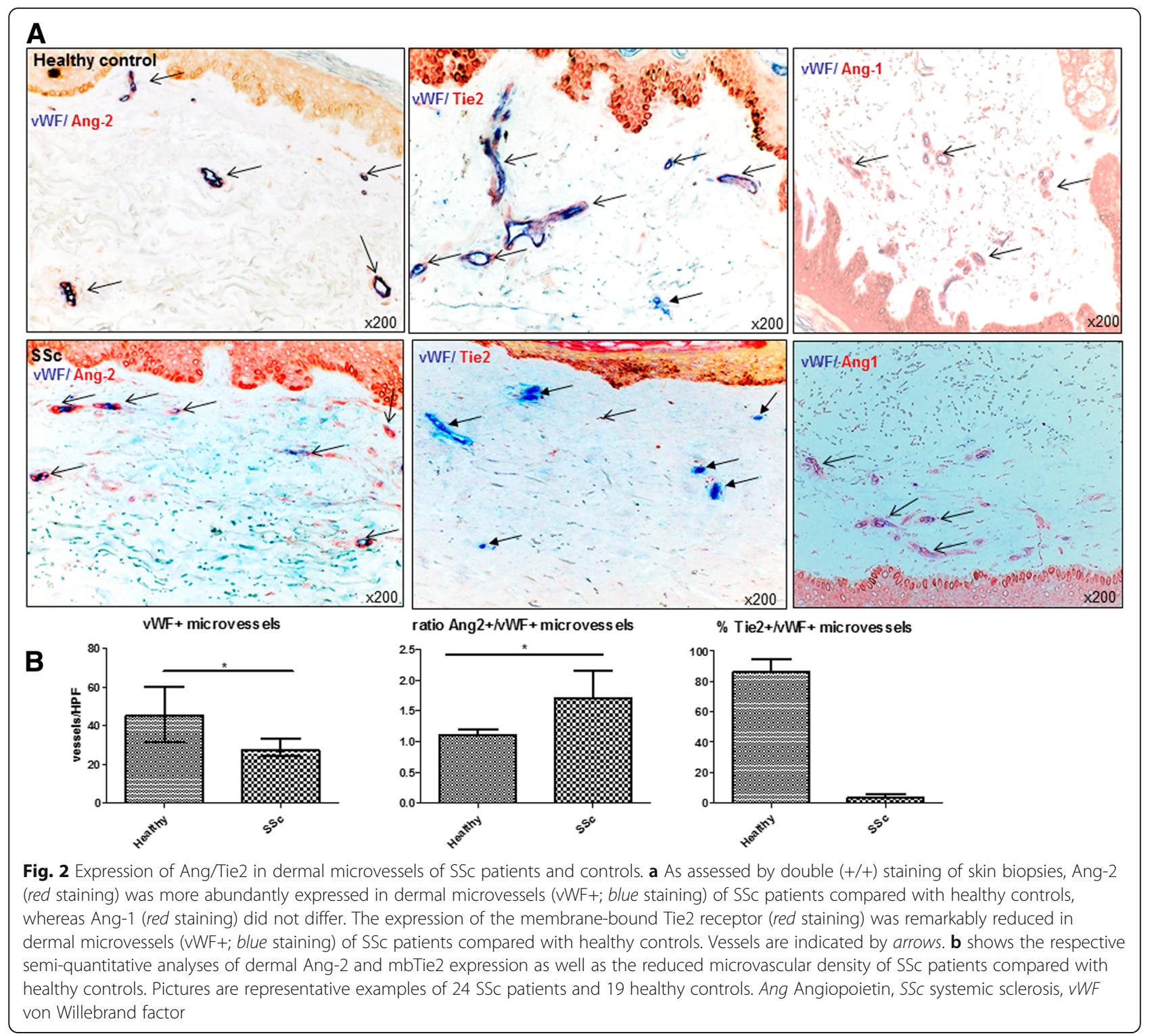

with increasing dosages of VEGF for $12 \mathrm{~h}$ reduced the numbers of Tie2-positive cells by $9.6,20.2$, and $25.1 \%$ whereas in parallel there was an increase of the sTie 2 concentration in the supernatants by 47 to $57 \%$ (Fig. 3b). Serum Ang-2 levels were increased in both lcSS and dcSSc patients as compared with healthy controls $(1.4 \pm 0.2$ and $1.5 \pm 0.3$ vs. $0.3 \pm 0.02 \mathrm{ng} / \mathrm{ml} ; p<0.01)$ whereas the Ang- 1 levels where remarkably lower $(18.4 \pm 5.7$ and $32.9 \pm 10.3$ vs. $68.4 \pm 5.4 \mathrm{ng} / \mathrm{ml} ; p<0.01$ ) (Fig. 3a). Irrespective of the cutaneous subtype, in the whole group of SSc patients with established disease, the serum levels of Ang-1 (23.9 \pm 5.3), Ang-2 (1.5 \pm 0.2$)$, and sTie2 (17.9 \pm 1.1$)$ differed significantly from healthy controls $(p<0.001$, respectively). The imbalance towards Ang-2 in patients with lcSSc and dcSSc resulted in a strongly reduced Ang-1/-2 ratio as compared with healthy controls (13:1 and $23: 1$ vs. 221:1, respectively; $p<0.05$. Interestingly, SSc patients with digital ulcers had a significantly lower Ang-1/Ang-2 ratio as compared with those without $\left(2.4_{(2,3)}\right.$ vs. $7.2_{(7,9)}$; $p<0.02)$. In contrast, patients with teleangiectasias had a significantly higher Ang-1/Ang- 2 ratio (1.9 \pm 0.4 vs. $1.3 \pm 0.08 ; p<0.05)$ than those without. Most importantly, in patients with pre-SSc, serum levels of Ang-1/-2 already differed significantly from healthy controls $(35.5 \pm$ 8.8 vs. $68.4 \pm 5.4 \mathrm{ng} / \mathrm{ml}$ and $0.9 \pm 0.2$ vs. $0.3 \pm 0.02 \mathrm{ng} / \mathrm{ml}$; $p<0.02$ ) (Fig. 3a). Serum levels of sTie2 were as high as in patients with established disease and thus remarkably elevated compared with healthy controls $(14.4 \pm 1.9$ vs. $9.6 \pm$ $1.8 \mathrm{ng} / \mathrm{ml} ; p=0.01$ ) (Fig. 3a). Of note, in line with previous results $[2,3]$, pre-SSc patients also had significantly elevated serum VEGF levels compared with healthy controls $\left(481_{(120,559)}\right.$ vs. $\left.101_{(10,169) ;} p=0.01\right)$, which were as high as 


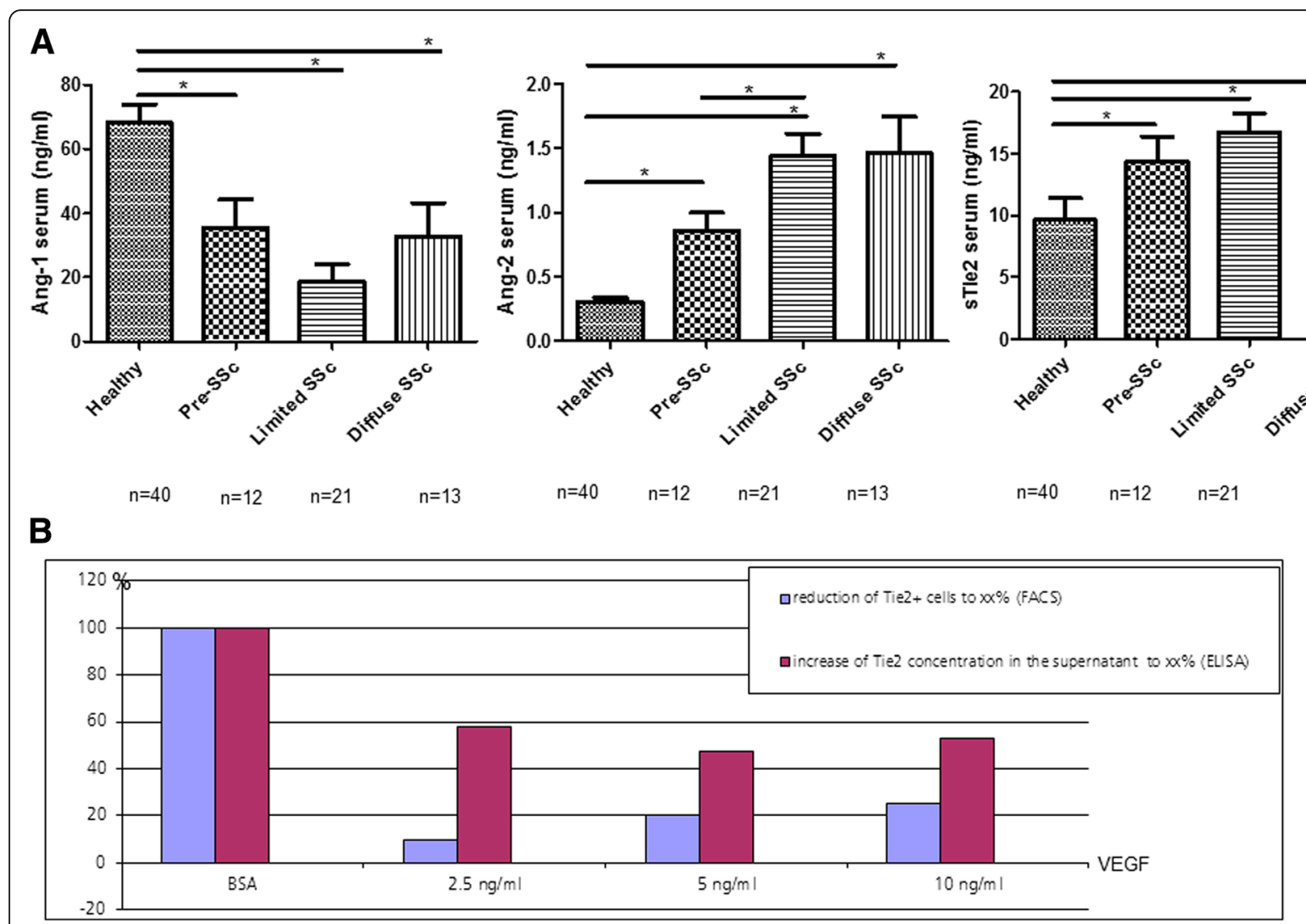

Fig. 3 Serum levels of Ang/Tie2 in SSC patients and controls and effects of VEGF on human dermal microvascular endothelial cells. a As assessed by ELISA, serum levels of Ang-2 were significantly increased in patients with pre-SSc as well as in established disease irrespective of the disease subset ( $\mid c=$ limited cutaneous vs. $d c=$ diffuse cutaneous) compared with healthy controls. In contrast, the Ang-1 levels were remarkably decreased in all different SSC subsets compared with healthy controls. Of note, all SSc patients irrespective of cutaneous subtype or disease stage showed elevated serum concentrations of sTie2 compared with healthy controls. b. Treatment of dermal HMVECs with recombined human VEGF led to a dose-dependent reduction of Tie2+ cells with concomitant increase of sTie2 in the supernatants thereby supporting the shedding hypothesis. Ang Angiopoietin, SSc systemic sclerosis, sTie2 soluble Tie2, VEGF vascular endothelial growth factor

in patients with established disease (lcSSc 412 $(250,609)$ and dcSSc 634 $(404,941) \mathrm{pg} / \mathrm{ml})$.

In summary, we observed a severe disturbance in the expression of Ang-1 and Ang-2 as well as of Tie2 in the skin and sera of patients SSc.

\section{Potential influences on the Ang/Tie2 expression in vivo}

To assess which pathogenic factors in SSc might affect the expression of Ang/Tie2, we evaluated pathophysiologically different animal models of SSc. First, we studied the Ang/Tie2 system in a vascular model of SSc, using the VEGF tg mice since VEGF plays a key role in SScassociated microvasculopathy and has been suggested to strongly influence the effects of Ang/Tie2 on vascular remodelling [6, 13] (Fig. 1). As previously shown, VEGF tg mice develop a proliferative vasculopathy with tortuous and dilated capillaries with increased vessel wall thickness reminiscent of both the capillary changes observed in SSc [1] and in Ang-2-overexpressing mice [11]. In VEGF+/+ tg mice as compared with VEGF+/- tg mice, chronically high levels of VEGF dampen the proangiogenic effects of VEGF [1], which is similarly observed in SSc patients with a chronically elevated VEGF levels and insufficient angiogenesis [2, 3].

In VEGF-+/- and VEGF $+/+$ tg mice, a higher proportion of dermal microvessels (vWF+) expressed Ang-2 compared with wt mice (\% of Ang-2+/vWF+ microvessels/HPF $100_{(78,100)}$ and $100_{(100,100)}$ vs. $36.7_{(0,57)} ; p=0.004, p=0.0003$ ) (Fig. 4a), whereas Ang1 was reduced (\% of Ang-1+/vWF+ microvessels/HPF $0_{(0,50)}$ and $20_{(0,50)}$ vs. $\left.75_{(66.7,75)} ; p=0.04, p=0.05\right)$ (Fig. 4b). Of note, expression of mbTie2 was remarkably reduced in the dermal capillaries ( $\mathrm{vWF}+$ ) of both $\mathrm{VEGF}+/$ - and VEGF+/+ tg mice as compared with controls (\% of Tie $2+/ \mathrm{vWF}+$ microvessels/HPF $50_{(25,100)}$ and $16.7_{(0,50)}$ vs. $100_{(88.3,100)} ; p=0.03, p=0.0006$ ) (Fig. 4c). These observations are in line with previous data showing that VEGF tips the balance towards Ang-2 and decreases the local expression of mbTie2 [27]. Furthermore, the decreased Ang-1/-2 ratio with a strong concomitant loss of 


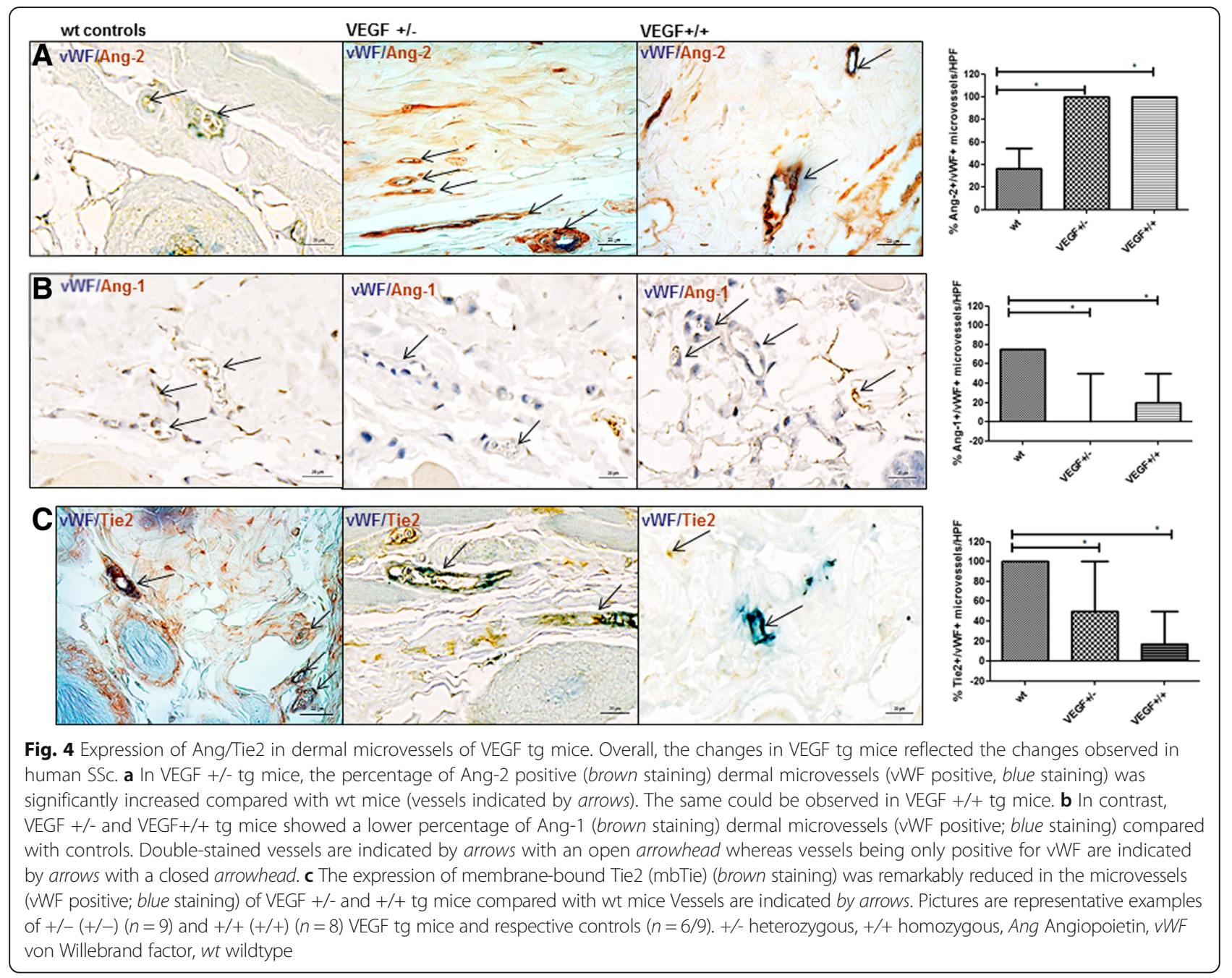

mbTie2 in chronic VEGF exposure in vivo mirrored the observed changes in SSc (Fig. 2).

Tissue hypoxia is a known phenomenon in SSc $[2,4]$. To evaluate whether hypoxia might contribute to the dysbalance of the Ang/Tie2 system in SSc, we analysed the tissue expression in hypoxic compared to normoxic mice. Whereas the expression of Ang-1 mRNA did not change, Ang-2 and Tie2 mRNA transcripts increased on the tissue level in hypoxia $(2.4 \pm 0.4$-fold; $p=0.027$ and $2.6 \pm 0.8$-fold; $p=0.042$ ) compared to normoxia (Additional file 2). Our data are in accordance with previous studies showing that hypoxia increases the expression of Ang-2 [6]. Since Ang-2 blocks Tie2 signalling, this ultimately leads to vessel destabilization and regression [6].

Since inflammation is another characteristic pathogenic feature in early stages of SSc, we next assessed the expression levels of Ang/Tie2 in the model of bleomycin-induced skin fibrosis [28] since it mimics early, inflammatory stages of SSc [29]. Treatment with bleomycin resulted in significant skin fibrosis as measured by increase in dermal thickness (mean \pm SEM $1.5 \pm 0.05$ fold) and hydroxyproline contents (1.6 \pm 0.1 -fold; $p<0.05$ each) (Additional file 3). Interestingly, there was a trend towards a higher percentage of Ang-2+ dermal microvessels $(\mathrm{vWF}+)$ in bleomycin-challenged mice vs. salinetreated controls (\% of Ang-2+/vWF+ microvessels/HPF $95.8 \pm 2.7$ vs. $65 \pm 15 ; p=0.08$ ) (Fig. 5a). No differences were observed for the expression of Ang- 1 in controls and bleomycin-treated mice (\% of Ang-1+/vWF+ microvessels/HPF $66^{7} 7_{(66.7,75)}$ vs. $\left.100_{(66.7,100)} ; p=0.3\right)$ (Fig. 5b). The expression of mbTie2 in dermal capillaries $(\mathrm{vWF}+)$ was lower in bleomycin-treated mice vs. saline-treated controls (\% of Tie2+/vWF+ microvessels/HPF $50_{(33,75)}$ vs. $100_{(94,100)} ; p=0.002$ ) (Fig. 5c). These observations are in line with recent data giving evidence of that in inflammatory conditions, the expression of Ang-2 is increased whereas the expression of mbTie2 is decreased [7].

Finally, we investigated the dermal expression of Ang/ Tie2 in an inflammation-independent, late-stage model 


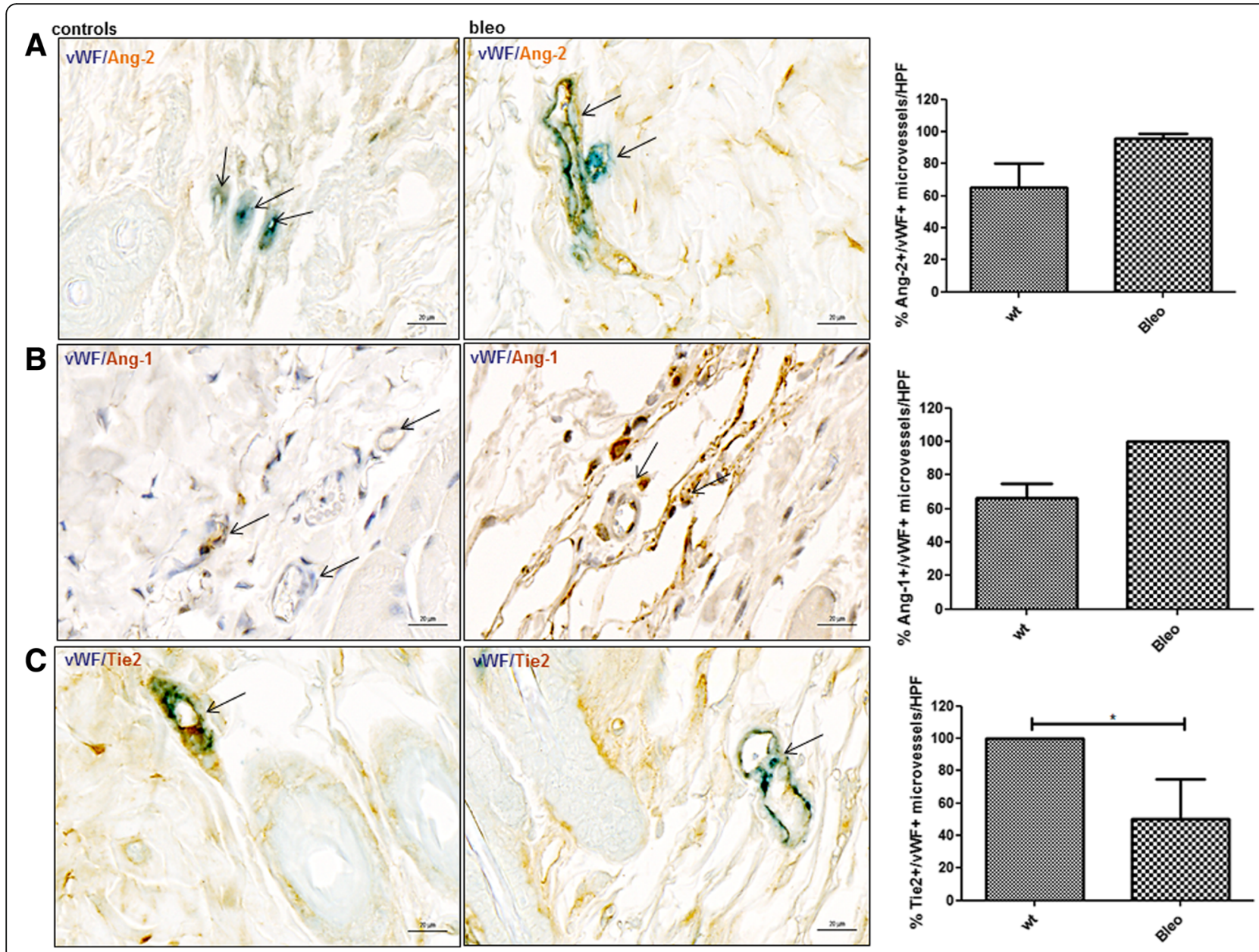

Fig. 5 Expression of Ang/Tie2 in dermal microvessels of bleomycin-challenged mice. $\mathbf{a}$, $\mathbf{b}$ In bleomycin-treated mice, no significant difference in the expression of Ang-2 or Ang-1 (brown staining) could be observed in dermal microvessels (vWF positive; blue staining) compared with saline-treated controls. Vessels are indicated by arrows. c The percentage of mbTie2 positive (brown staining) dermal microvessels (vWF positive; blue staining) was significantly higher in bleomycin- vs. saline-treated mice. Vessels are indicated by arrows. Pictures are representative examples of four bleomycin-treated and four saline-treated controls. Ang Angiopoietin, HPF high power field, vWF von Willebrand factor, wt wildtype

of SSc, the TSK1 model [30]. In TSK1 mice, the expression of Ang-2 in capillaries (vWF+) was not changed compared with pa/pa controls $\left(100_{(50,100)}\right.$ vs. $100_{(88,100)}$; $p=0.9$ ) (Fig. 6a). No statistically significant difference was observed in TSK1 mice vs. pa/pa controls neither for Ang-1 (\% of Ang-1+/vWF+ microvessels/HPF $0_{(0,25)}$ vs. $2_{(0,75)} ; p=0.2$ ) nor for mbTie2 (\% of Tie2+/vWF+ microvessels/HPF $58.4_{(13,67)}$ vs. $100_{(0,100)}, p=0.2$ ) (Fig. 6b, c) although there was a tendency to a reduction of Tie2 + microvessels in TSK1 mice.

\section{Discussion}

In SSc, peripheral microvasculopathy is a key pathogenic event. Our results suggest that a dysbalance of Ang/Tie2 might contribute to the vascular changes. An excess of Ang-2 over Ang-1 - as herein observed on tissue and serum level - is known to induce vascular remodelling by blocking Tie2 signalling on EC [6, 10, 11] (Fig. 1). Interestingly, SSc patients with digital ulcers showed a significantly lower Ang-1/-2 ratio as compared with those without, which further underlines the potential clinical relevance of the observed alterations although the low number of patients and the heterogeneity of the disease warrant some caution in the interpretation of these results. Depending on the absence/presence of VEGF [6, 13], the destabilization of vessels might then lead to regression or (disorganized) sprouting (Fig. 1), both well-described events in SSc microvasculopathy. Moreover, the substantial loss of mbTie2 on dermal microvessels in SSc with concomitant increase in sTie2, which also competes with Ang-1, further impairs Tie2 signalling (Fig. 1). Notably, treatment of dermal HMVECs with VEGF led to a dose-dependent reduction of Tie $2+$ cells with concomitant increase of sTie2 in 


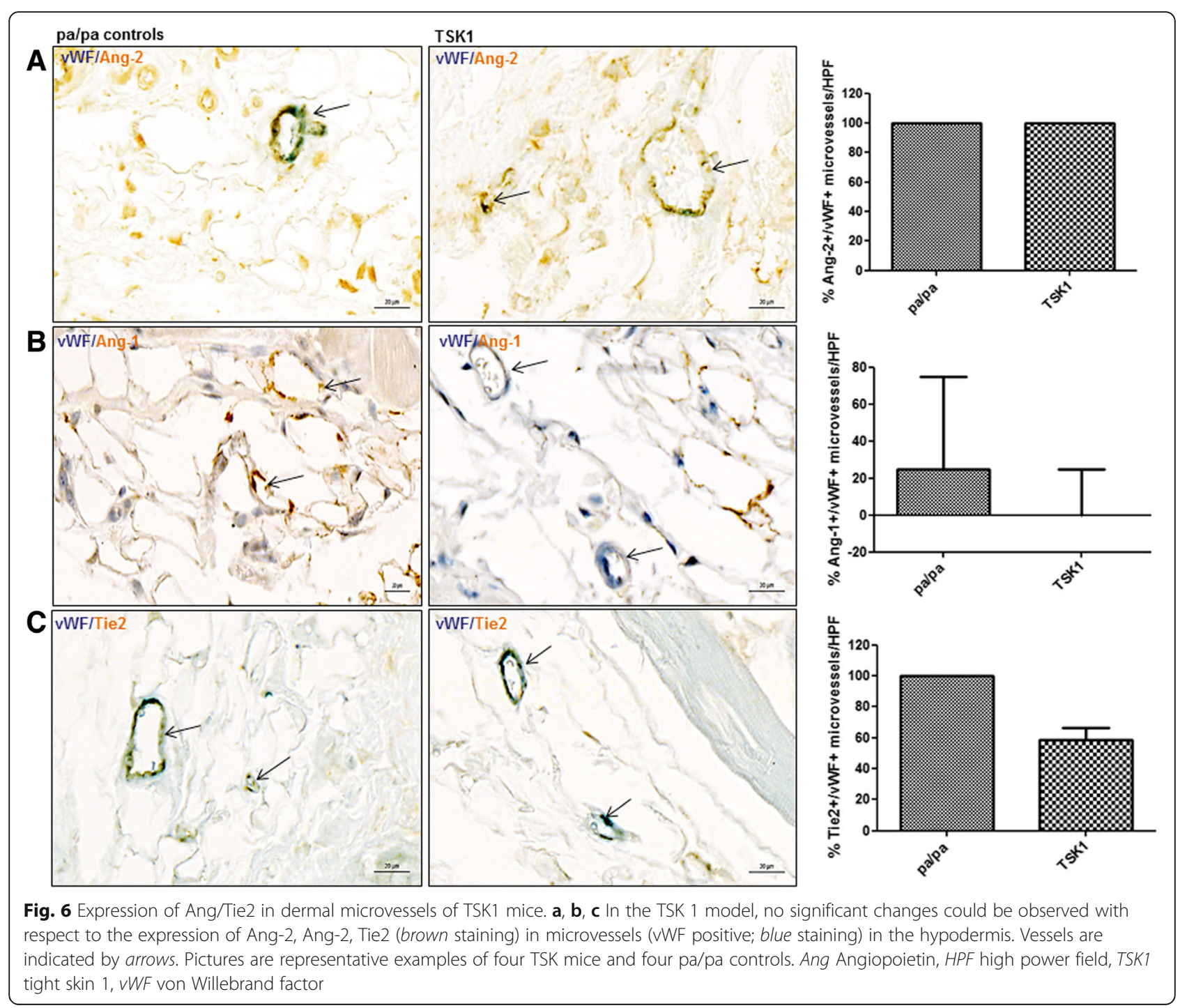

the supernatants thereby supporting the shedding hypothesis. Interestingly, a similar dysbalance of Ang-1/-2, sTie2, and VEGF levels was already observed in pre-SSc patients as in patients with established disease indicating that the disturbance of angiogenic signalling networks is an early pathogenic event.

Our data on the expression of angiopoietins and Tie2 in dermal microvessels of SSc patients extend previous, partially conflicting results on the systemic levels of Ang-1/-2 and sTie2 in SSc. Interestingly, all available studies [16, 31-33] as well as our own data found increased systemic levels of Ang-2 without differences regarding lcSSc or dcSSc subtypes [32, 33]. Data on Ang-1 are more ambiguous. Whereas Michalska et al. reported a similar reduction of Ang-1 levels and the Ang-1/Ang-2 ratio in SSc [16], the study by Dunne et al. showed an increase of Ang-1 in the dcSSc subset, but not the combined SSc group as compared with healthy controls [32]. However, apart from discrepancies that might arise from studying such a heterogeneous disease, these differences might also be attributed to the use of plasma [32] instead of serum. Similar to our observations, the serum levels of sTie2 were found to be elevated by Noda et al [17] and Dunne et al. [32], however, the latter study only demonstrating significant differences in the lcSSc subgroup. The study by Gerlicz et al. showed a trend towards higher levels of sTie2 in both SSc subsets compared with healthy controls, yet the data were not statistically significant [33]. But again, all these studies including our own have only analysed very limited numbers of patients. Therefore, the observed changes of serum/plasma angiopoietin and sTie2 levels in SSc and particularly their correlation with clinical (vascular) features warrant a standardized and systematic evaluation in a large, multicentre cohort. Apart from the fact that in most published studies not all three parameters (Ang-1, Ang-2, sTie2) have been 
simultaneously analysed, some of the current discrepancies of the association with clinical vascular features might arise from differences such as treatment (vasodilating agents, immunosuppressive drugs), the definitions of ulcers and nailfold capillaroscopy patterns used, but also biobank-related issues such as different processing and/or storage of blood samples and use of different ELISA assays. Thus, although the current available data support a dysbalance of the Ang/Tie2 system in SSc, the clinical implications have yet to be elucidated including the performance of more detailed functional in vitro experiments.

Our in vivo data derived from the different animal models, which closely mirrored the changes observed in SSc patients, suggested that VEGF, hypoxia, and inflammation might play a role in the dysbalance of the Ang/ Tie2 system in SSc.

\section{Conclusions}

Thus, our data extend the previous concept of peripheral microangiopathy in SSc $[1-4,34]$ giving novel evidence on tissue level of an even more complex dysregulation of angiogenic key players. The dysregulation of the Ang/Tie-2 system appears to be specific for vascular and inflammatory conditions, as the non-vascular and inflammation-independent TSK1 model did not reflect the changes observed in the human disease. This further underlines that for proof of concept studies the appropriate choice of an animal model that closely reflects the situation of the human disease, is of outmost importance [35]. However, for the development of targeted treatment strategies those interactions and their biologic relevance will first have to be carefully and thoroughly studied in suitable preclinical settings including detailed functional experiments and specific animal models.

\section{Additional files}

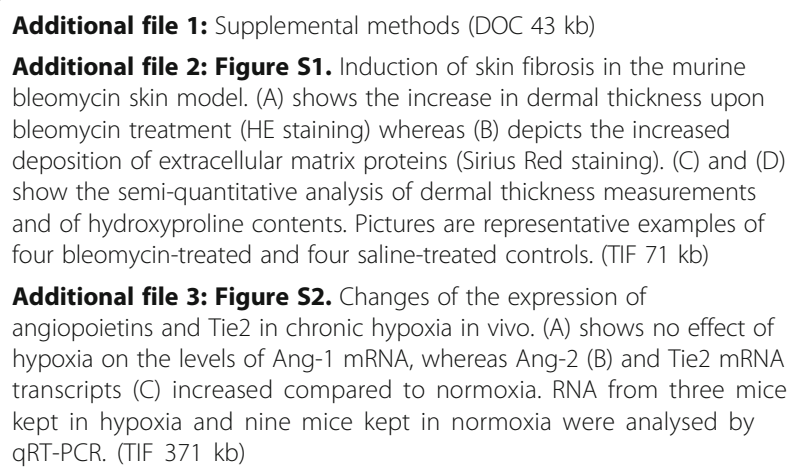

Additional file 3: Figure S2. Changes of the expression of angiopoietins and Tie2 in chronic hypoxia in vivo. (A) shows no effect of hypoxia on the levels of Ang-1 mRNA, whereas Ang-2 (B) and Tie2 mRNA transcripts (C) increased compared to normoxia. RNA from three mice kept in hypoxia and nine mice kept in normoxia were analysed by qRT-PCR. (TIF $371 \mathrm{~kb}$ )

\section{Abbreviations}

+/-: Heterozygous; +/+: Homozygous; Ang: Angiopoietin; dc: Diffuse cutaneous; EC: Endothelial cells; HMVEC: Human microvascular endothelial cell; HPF: High power field; IHC: Immunohistochemistry; lc: Limited cutaneous; mb: Membrane bound; SEM: Standard error of the mean; SSc: Systemic sclerosis; sTie2: Soluble Tie2; tg: Transgenic; TSK1: Tight skin 1; VEGF: Vascular endothelial growth factor; VWF: von Willebrand factor; wt: Wildtype

\section{Acknowledgements}

The authors thank Maria Comazzi for the excellent technical support. Imaging was performed with a slide scanner (Zeiss Axio Scan.Z1, software: Zen lite, blue edition 2.3) provided and maintained by the Center for Microscopy and Image Analysis, University of Zurich.

\section{Availability of data and materials}

All data generated or analysed during this study are included in this published article and its supplementary information files.

\section{Authors' contributions}

FM has made substantial contributions to the conception of the study and the acquisition, analysis and the interpretation of data and was involved in drafting the manuscript. JS was centrally involved in the acquisition and analysis of data and in revising the manuscript. JHWD, RG, and SG made contributions to the conception and design of the study, in the interpretation of the data and the revision of the manuscript. OD was involved in the conception and design of the study, the interpretation of data and in drafting and revising the manuscript. BM made substantial contributions to conception and design of the study, was centrally involved in the acquisition, analysis and interpretation of data, and in drafting and revising the manuscript. All authors have given final approval of the version to be published.

\section{Competing interests}

FM had Articulum Fellowship program (Pfizer), congress funding from Roche, Lilly, Medac

JHWD has consultancy relationships and/or has received research funding from Actelion, Anamar, BMS, Celgene, Bayer Pharma, Boehringer Ingelheim, JB Therapeutics, Sanofi-Aventis, Novartis, UCB, GSK, Array Biopharma and Active Biotech in the area of potential treatments of SSC and is stock owner of $4 D$ Science. OD had a consultancy relationship and/or has received research funding from 4 D Science, Actelion, Active Biotec, Chemom AG, Bayer, Biogenldec, BMS, Boehringer Ingelheim, ChemomAb, EpiPharm, espeRare foundation, Genentech/Roche, GSK, Inventiva, Lilly, medac, Medlmmune, Pharmacyclics, Pfizer, Sanofi, Serodapharm, and Sinoxa in the area of potential treatments of scleroderma and its complications. In addition, Patent licensed: mir-29 for the treatment of systemic sclerosis. Speaker's fees from AbbVie, iQone Healthcare, and Mepha.

BM had grant/research support from AbbVie, Protagen, EMDO, Novartis, congress support from Pfizer, Roche, and Actelion. Patent licensed: mir-29 for the treatment of systemic sclerosis.

All others were supported by their respective institutions.

\section{Consent for publication}

Not applicable.

\section{Ethics approval and consent to participate}

All participants had signed a consent form approved by the local institutional review boards. The study had been approved by the cantonal ethics committee Zurich. The animal protocols were approved by the cantonal veterinary office Zurich.

\section{Publisher's Note}

Springer Nature remains neutral with regard to jurisdictional claims in published maps and institutional affiliations.

\section{Author details}

'Department of Rheumatology, University Hospital Zurich, Gloriastrasse 25, 8091 Zurich, Switzerland. 'Department of Oncology, St. Georg Hospital, Leipzig, Germany. ${ }^{3}$ Department of Internal Medicine 3, University Hospital, Erlangen, Germany. 
Received: 29 November 2016 Accepted: 2 May 2017 Published online: 25 May 2017

\section{References}

1. Maurer B, Distler A, Suliman YA, Gay RE, Michel BA, Gay S, Distler JH, Distler O. Vascular endothelial growth factor aggravates fibrosis and vasculopathy in experimental models of systemic sclerosis. Ann Rheum Dis. 2014;73(10):1880-7.

2. Distler O, Distler JH, Scheid A, Acker T, Hirth A, Rethage J, Michel BA, Gay RE, Muller-Ladner U, Matucci-Cerinic M, et al. Uncontrolled expression of vascular endothelial growth factor and its receptors leads to insufficient skin angiogenesis in patients with systemic sclerosis. Circ Res. 2004;95(1):109-16.

3. Distler O, Del Rosso A, Giacomelli R, Cipriani P, Conforti ML, Guiducci S, Gay RE, Michel BA, Bruhlmann P, Muller-Ladner U, et al. Angiogenic and angiostatic factors in systemic sclerosis: increased levels of vascular endothelial growth factor are a feature of the earliest disease stages and are associated with the absence of fingertip ulcers. Arthritis Res. 2002;4(6):R11.

4. Distler JH, Jungel A, Pileckyte M, Zwerina J, Michel BA, Gay RE, KowalBielecka O, Matucci-Cerinic M, Schett G, Marti HH, et al. Hypoxia-induced increase in the production of extracellular matrix proteins in systemic sclerosis. Arthritis Rheum. 2007;56(12):4203-15.

5. Distler JH, Gay S, Distler O. Angiogenesis and vasculogenesis in systemic sclerosis. Rheumatology. 2006;45 Suppl 3:iii26-27.

6. Thomas M, Augustin HG. The role of the Angiopoietins in vascular morphogenesis. Angiogenesis. 2009;12(2):125-37.

7. Milam KE, Parikh SM. The angiopoietin-Tie2 signaling axis in the vascular leakage of systemic inflammation. Tissue Barriers. 2015;3(1-2):e957508.

8. Scholz A, Plate KH, Reiss Y. Angiopoietin-2: a multifaceted cytokine that functions in both angiogenesis and inflammation. Ann N Y Acad Sci. 2015;1347:45-51.

9. Vajkoczy P, Farhadi M, Gaumann A, Heidenreich R, Erber R, Wunder A, Tonn JC, Menger MD, Breier G. Microtumor growth initiates angiogenic sprouting with simultaneous expression of VEGF, VEGF receptor-2, and angiopoietin-2. J Clin Invest. 2002;109(6):777-85.

10. Scharpfenecker M, Fiedler U, Reiss Y, Augustin HG. The Tie-2 ligand angiopoietin-2 destabilizes quiescent endothelium through an internal autocrine loop mechanism. J Cell Sci. 2005;118(Pt 4):771-80.

11. Maisonpierre PC, Suri C, Jones PF, Bartunkova S, Wiegand SJ, Radziejewski C, Compton D, McClain J, Aldrich TH, Papadopoulos N, et al. Angiopoietin-2, a natural antagonist for Tie2 that disrupts in vivo angiogenesis. Science. 1997;277(5322):55-60.

12. Etoh T, Inoue H, Tanaka S, Barnard GF, Kitano S, Mori M. Angiopoietin-2 is related to tumor angiogenesis in gastric carcinoma: possible in vivo regulation via induction of proteases. Cancer Res. 2001;61(5):2145-53.

13. Findley CM, Cudmore MJ, Ahmed A, Kontos CD. VEGF induces Tie2 shedding via a phosphoinositide 3-kinase/Akt dependent pathway to modulate Tie2 signaling. Arterioscler Thromb Vasc Biol. 2007;27(12):2619-26.

14. Reusch P, Barleon B, Weindel K, Martiny-Baron G, Godde A, Siemeister G, Marme D. Identification of a soluble form of the angiopoietin receptor TIE-2 released from endothelial cells and present in human blood. Angiogenesis. 2001:4(2):123-31.

15. Makinde T, Agrawal DK. Intra and extravascular transmembrane signalling of angiopoietin-1-Tie2 receptor in health and disease. J Cell Mol Med. 2008;12(3):810-28.

16. Michalska-Jakubus M, Kowal-Bielecka O, Chodorowska G, Bielecki M, Krasowska D. Angiopoietins-1 and -2 are differentially expressed in the sera of patients with systemic sclerosis: high angiopoietin-2 levels are associated with greater severity and higher activity of the disease. Rheumatology (Oxford). 2011;50(4):746-55

17. Noda S, Asano Y, Aozasa N, Akamata K, Yamada D, Masui Y, Tamaki Z, Kadono T, Sato S. Serum Tie2 levels: clinical association with microangiopathies in patients with systemic sclerosis. J Eur Acad Dermatol Venereol. 2011; 25(12):1476-9

18. Yuan HT, Khankin EV, Karumanchi SA, Parikh SM. Angiopoietin 2 is a partial agonist/antagonist of Tie2 signaling in the endothelium. Mol Cell Biol. 2009;29(8):2011-22.

19. LeRoy EC, Black C, Fleischmajer R, Jablonska S, Krieg T, Medsger Jr TA, Rowell N, Wollheim F. Scleroderma (systemic sclerosis): classification, subsets and pathogenesis. J Rheumatol. 1988;15(2):202-5.
20. Cutolo M, Sulli A, Pizzorni C, Accardo S. Nailfold videocapillaroscopy assessment of microvascular damage in systemic sclerosis. J Rheumatol. 2000;27(1):155-60

21. Detmar M, Brown LF, Schon MP, Elicker BM, Velasco P, Richard L, Fukumura D, Monsky W, Claffey KP, Jain RK. Increased microvascular density and enhanced leukocyte rolling and adhesion in the skin of VEGF transgenic mice. J Invest Dermatol. 1998;111(1):1-6.

22. Akhmetshina ADC, Pileckyte M, Maurer B, Axmann R, Jüngel A, Zwerina J, Gay S, Schett G, Distler O, Distler JH. Dual inhibition of c-abl and PDGF receptor signaling by dasatinib and nilotinib for the treatment of dermal fibrosis. FASEB J. 2008:22:2214-22.

23. Siracusa LD, McGrath R, Ma Q, Moskow JJ, Manne J, Christner PJ, Buchberg AM, Jimenez SA. A tandem duplication within the fibrillin 1 gene is associated with the mouse tight skin mutation. Genome Res. 1996;6(4):300-13.

24. Distler JHJA, Huber LC, Schulze-Horsel U, Zwerina J, Gay RE, Michel BA, Hauser T, Schett G, Gay S, Distler O. Imatinib mesylate reduces production of extracellular matrix and prevents development of experimental dermal fibrosis. Arthritis Rheum. 2007;56:311-22.

25. Distler JH, Jungel A, Huber LC, Schulze-Horsel U, Zwerina J, Gay RE, Michel BA, Hauser T, Schett G, Gay S, et al. Imatinib mesylate reduces production of extracellular matrix and prevents development of experimental dermal fibrosis. Arthritis Rheum. 2007;56(1):311-22

26. Ball SG, Shuttleworth CA, Kielty CM. Vascular endothelial growth factor can signal through platelet-derived growth factor receptors. J Cell Biol. 2007; 177(3):489-500.

27. Findley CM, Cudmore MJ, Ahmed A, Kontos CD. VEGF induces Tie2 shedding via a phosphoinositide 3-kinase/Akt-dependent pathway to modulate Tie2 signaling. Arterioscler Thromb Vasc Biol. 2007;27(12):2619-26.

28. Akhmetshina A, Venalis P, Dees C, Busch N, Zwerina J, Schett G, Distler O, Distler JH. Treatment with imatinib prevents fibrosis in different preclinical models of systemic sclerosis and induces regression of established fibrosis. Arthritis Rheum. 2009;60(1):219-24.

29. Beyer C, Schett G, Distler O, Distler JH. Animal models of systemic sclerosis: prospects and limitations. Arthritis Rheum. 2010;62(10):2831-44

30. Green MC, Sweet HO, Bunker LE. Tight-skin, a new mutation of the mouse causing excessive growth of connective tissue and skeleton. Am J Pathol. 1976:82(3):493-512.

31. Riccieri V, Stefanantoni K, Vasile M, Macri V, Sciarra I, lannace N, Alessandri C, Valesini G. Abnormal plasma levels of different angiogenic molecules are associated with different clinical manifestations in patients with systemic sclerosis. Clin Exp Rheumatol. 2011;29(2 Suppl 65):S46-52.

32. Dunne JV, Keen KJ, Van Eeden SF. Circulating angiopoietin and Tie-2 levels in systemic sclerosis. Rheumatol Int. 2013;33(2):475-84.

33. Gerlicz Z, Dziankowska-Bartkowiak B, Dziankowska-Zaborszczyk E, Sysa-Jedrzejowska A. Disturbed balance between serum levels of receptor tyrosine kinases Tie-1, Tie-2 and angiopoietins in systemic sclerosis. Dermatology. 2014;228(3):233-9.

34. Manetti M, Guiducci S, Romano E, Ceccarelli C, Bellando-Randone S, Conforti ML, Ibba-Manneschi L, Matucci-Cerinic M. Overexpression of VEGF165b, an inhibitory splice variant of vascular endothelial growth factor, leads to insufficient angiogenesis in patients with systemic sclerosis. Circ Res. 2011;109(3):e14-26.

35. Maurer B, Distler A, Dees C, Khan K, Denton CP, Abraham D, Gay RE, Michel BA, Gay S, Hw Distler J, et al. Levels of target activation predict antifibrotic responses to tyrosine kinase inhibitors. Ann Rheum Dis. 2013;72(12):2039-46. 